\title{
IL MALADETTO FIORE: DANTE E IL DENARO ALLE ORIGINI DEL CAPITALISMO ${ }^{1}$
}

\section{Il maladetto fiore: Dante and Money at the Onset of Capitalism}

\author{
Donatella Stocchi-Perucchio \\ University of Rochester
}

\section{Sommario}

La riflessione di Dante sul denaro è un aspetto rilevante della sua filosofia morale e politica e motivo centrale nel suo immaginario poetico. La Firenze del tempo aveva una potente classe borghese e una moneta tra le più prestigiose d'Europa e Dante, da fiorentino, fu testimone dell'insorgere di un'economia di profitto i cui rischi morali non mancò di rilevare. Questo saggio propone una riflessione su come l'atteggiamento di Dante in merito al denaro si rapporti ad alcuni aspetti del pensiero di Emmanuel Levinas e Michel Henry, due filosofi che criticano le economie capitaliste occidentali e ribadiscono l'importanza della prospettiva umanistica nell'analisi delle realtà economiche.

\section{Parole chiave}

Denaro; avarizia; usura; falsificazione; ricchezza

\begin{abstract}
Dante's concern with money is a significant aspect of his moral and political philosophy and a central motif in his poetic imagination. As a citizen of Florence, a city with a powerful bourgeois class and a currency among the most prestigious of Europe, Dante witnessed the onset of a profit economy and did not fail to see its moral risks. This essay reflects on how Dante's stance on the issue of money can be related to points made by Emmanuel Levinas and Michel Henry, who are critics of western capitalist economies and who insist that economic realities must be analyzed from a humanistic perspective.
\end{abstract}

${ }^{1}$ Una versione analoga del presente saggio sarà pubblicata in lingua inglese entro il 2020 negli Atti del Convegno «Pensare el dinero», tenutosi all'Università del Pacifico di Lima, Perù, nel 2015, editi da J. Wiesse Rebagliati. 


\section{Keywords}

Money; avarice; usury; counterfeit; wealth

L'idea di pensare filosoficamente il denaro, secondo l'espressione di Michel Henry, ${ }^{2}$ non potrebbe essere più congeniale a Dante il cui interesse per i temi del denaro e dell'economia costituisce uno dei tratti più salienti della sua filosofia morale e politica, come pure uno dei motivi centrali del suo immaginario poetico. ${ }^{3}$ A partire dalle prime canzoni fino al Convivio, alla Monarchia e alla Commedia, Dante non si stanca di rivisitare questi temi. Da cittadino di Firenze, città con una classe borghese potente e una moneta tra le più prestigiose d'Europa, Dante fu testimone dell'insorgere di un'economia di profitto mercantile e protocapitalista della quale non mancò di rilevare i rischi morali e il potenziale distruttivo. In queste pagine rifletterò su come l'atteggiamento di Dante in merito al denaro si possa confrontare con il punto di vista di due fenomenologi moderni, Emmanuel Levinas (1906-1995) e Michel Henry (1922-2002), attenti osservatori e critici delle economie capitaliste occidentali. Essi sostengono infatti che i fenomeni economici siano da analizzare, e auspicabilmente correggere, con le intuizioni che sole possono derivare dalla prospettiva umanistica. Ciò che io vedo nel loro metodo è un rivolgersi all'antico, in particolare ad Aristotele: un bisogno cioè di re-inscrivere l'economia nell'ambito dell'etica e nel contesto della giustizia.

In questi famosi versi dell'Inferno, Dante critica aspramente i nuovi ricchi di Firenze,

«La gente nuova e i sùbiti guadagni

orgoglio e dismisura han generata,

Fiorenza, in te, sì che tu già ten piagni». ${ }^{4}$

(If XVI 73-75, corsivo mio)

Nel canto XVI del Paradiso-il canto corrispondente nella terza cantica-egli descrive, per bocca del trisavolo Cacciaguida, la mobilità sociale come un'altra forma di eccesso, dannosa al benessere del corpo politico quanto la gola lo è, come apprendiamo dal canto di Ciacco, per il corpo fisico:

\footnotetext{
${ }^{2}$ Henry, M., «Comment penser l'argent?», in R.-P. Droit (a cura di), Troisième Forum Le Monde/Le Mans, Paris, Le Monde Editions, 1992, pp. 73-80.

${ }^{3}$ Basti pensare all'origine economica del concetto di contrapasso, di derivazione aristotelicotomista e alla sua importanza nella rappresentazione e riflessione dantesca sulla giustizia. Si veda Mazzotta, G., Dante's Vision and the Circle of Knowledge, Princeton, Princeton University Press, 1993, pp. 78-86; Armour, P., «Gold, Silver, and True Treasure. Economic Imagery in Dante», Romance Studies: A Journal of the University of Wales, 23 (1994), pp. 7-30. a p. 14.

${ }^{4}$ Tutte le citazioni dai testi danteschi sono tratte da Dante Alighieri, Le opere di Dante., F. Brambilla Ageno et al. (a cura di), Firenze, Società Dantesca Italiana, Polistampa, 2012.
} 


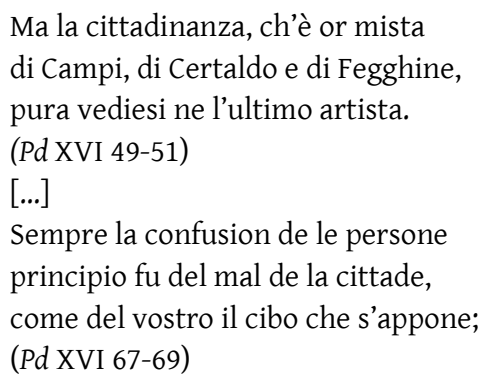

Qui Dante non esprime un astratto principio di ostilità per 'il diverso', ma si riferisce a persone specifiche le cui malefatte, come l'abuso d'ufficio, avevano alterato la vita politica di Firenze portando come conseguenza alla sconfitta dei Guelfi Bianchi e, con essa, al proprio esilio ed esclusione dall'amnistia del $1311 .^{5}$ Tuttavia, anche se leggiamo questi come risentimenti dettati da motivazioni personali visto che Dante stesso era stato accusato di abuso d'ufficio, sta di fatto che, in contrasto con l'attuale degenerazione della città, l'evocazione nostalgica dell'antica Firenze da parte di Cacciaguida testimonia inequivocabilmente l'atteggiamento conservatore di Dante verso quei mutamenti socioeconomici quali ad esempio l'avvento della classe media ovvero della borghesia urbana, l'incremento del commercio e la crescente economia di mercato. Dal suo punto di vista, tali mutamenti stimolavano la corsa al denaro e provocavano la destabilizzazione della famiglia, il culto del superfluo, la vanità, l'ostentazione e la competitività: ${ }^{6}$

Fiorenza dentro da la cerchia antica, ond'ella toglie ancora e terza e nona, si stava in pace, sobrïa e pudica.

Non avea catenella, non corona, non gonne contigiate, non cintura che fosse a veder più che la persona.

${ }^{5}$ Tale atteggiamento può apparire ripugnante se lo prendiamo come una forma di razzismo verso 'gli altri', cioè coloro che, arrivando in città dalle campagne, hanno contaminato la 'purezza' di sangue della popolazione urbana. Questo è il parere di F. Forlenza, magistrato e critico letterario che guarda a Dante dal punto di vista del criminologo. Cfr. Forlenza, F., Il diritto penale nella Commedia. Le radici del «sorvegliare e punire» nell'Occidente, Roma, Armando, 2003, p. 13. In realtà, Dante attacca qui individui ben noti per la loro influenza politica e per la loro baratteria come Baldo d'Aguglione e Fazio da Signa (Pd XVI 52-57). Cfr. Compagni, D., Cronica delle cose occorrenti ne' tempi suoi II 23, p. 66, http://www.letteraturaitaliana.net/pdf/Volume_1/t9.pdf. Data di consultazione 14/11/2019.

${ }^{6}$ Questo è ciò che Mark Block identifica con la seconda era feudale e altri storici considerano rivoluzione commerciale. Cfr. Little, L.K., «Introduction», in Religious Poverty and the Profit Economy in Medieval Europe, Ithaca, Cornell University Press, 1978, p. x. 
Non faceva, nascendo, ancor paura la figlia al padre, ché 'l tempo e la dote non fuggien quinci e quindi la misura.

Non avea case di famiglia vote; non v'era giunto ancor Sardanapalo a mostrar ciò che 'n camera si puote. Non era vinto ancora Montemalo dal vostro Uccellatoio, che, com'è vinto nel montar sù, così sarà nel calo.

Bellincion Berti vid'io andar cinto di cuoio e d'osso, e venir da lo specchio la donna sua sanza 'l viso dipinto; e vidi quel d'i Nerli e quel del Vecchio esser contenti a la pelle scoperta, e le sue donne al fuso e al pennecchio. Oh fortunate! ciascuna era certa de la sua sepultura, e ancor nulla era per Francia nel letto diserta.

(Pd XV 97-120, corsivo mio)

Se leggiamo queste pagine prima di tutto da una prospettiva filosofica, il concetto chiave è la dismisura, l'eccesso contrario alla virtù che, seguendo l'etica aristotelica del giusto mezzo, Dante condanna in tutta la sua opera. Il principio espresso nella canzone Doglia mi reca-"Come con dismisura si rauna, / così con dismisura si ristrigne; / e questo è quel che pigne / molti in servaggio» (1l. 85-88)-lo si trova riecheggiato sia nell' Inferno che nel Purgatorio, quando Dante tratta dell'avarizia, della prodigalità e dello sperpero. ${ }^{7} \mathrm{Nel}$ canto VII dell'Inferno dedicato all'economia e al ruolo della Fortuna nel distribuire i beni del mondo, gli avari e i prodighi «che con misura nullo spendio fêrci» (If VII 42), occupano gli estremi che delimitano il giusto atteggiamento verso il denaro ovvero lo spendere con misura.

Nel canto XXII del Purgatorio, il poeta Stazio, che ha appena completato la sua purgazione per il peccato di prodigalità-«e questa dismisura / migliaia di lunari hanno punita» (Pg XXII 35-36)-ha il compito di esaltare il valore della moderazione attraverso la distorsione di un verso virgiliano scritto originariamente per condannare l'avarizia: "Per che non reggi tu, o sacra fame / de l'oro, l'appetito de' mortali?'»(Pg XXII 40-41). La lettura in positivo di Stazio è resa possibile dal significato ambivalente della parola latina sacra nel doppio senso di sacra ed esecranda, e gli serve per attribuire all'intuizione morale di Virgilio il merito della

${ }^{7}$ Sulla tradizione dietro al concetto dantesco di misura cfr. Barolini, T., «Aristotle's Mezzo, Courtly Misura, and Dante's Canzone Le dolci rime. Humanism, Ethics, and Social Anxiety», in J. Ziolkowski (a cura di), Dante and the Greeks, Washington DC, Dumbarton Oaks, 2014, pp. 163-179. 
propria salvezza. Una dose di attaccamento alla ricchezza e alla proprietà che non dovrebbe essere dissipata senza ritegno, o peggio, sperperata come fanno gli scialacquatori condannati al settimo cerchio dell'Inferno, caratterizza il comportamento virtuoso che Dante chiama ne Le dolci rime, secondo l'Etica aristotelica, «un abito eligente / lo qual dimora in mezzo solamente» (vv. 86-87). La stessa idea sarà reiterata al centro della teoria dell'amore e del liberum arbitrium esposta da Virgilio nel Purgatorio: «Mentre ch'elli (l'amore naturale) è nel primo ben diretto, / e ne' secondi sé stesso misura, / esser non può cagion di mal diletto» (Pg XVII 97-99). È tuttavia certo che Dante veda l'avarizia non solo come l'estremo opposto della prodigalità, ma anche come un peccato molto più abominevole e pericoloso della prodigalità stessa, un fatto evidenziato fin dall'inizio della conversazione purgatoriale con Stazio dall'incompatibilità di questo vizio con la saggezza del poeta-«come poté trovar dentro al tuo seno / loco avarizia, tra cotanto senno / di quanto per tua cura fosti pieno?» (Pg XXII 22-24). Ciò si spiega perché, nella morale cristiana che Dante integra con quella aristotelica e rivisita, come vedremo, alla luce della nuova economia del denaro, l'avarizia ha il rango di peccato capitale. L'avarizia è più prona ad essere generata dalla ricchezza ed è significativo che Dante, sia che parli della moneta fiorentina, delle ricchezze in generale, o dell'avarizia, usi nei vari contesti lo stesso aggettivo maladetto. Riprova di questo è il canto IX del Paradiso in cui Dante considera Firenze e la sua ricchezza responsabili per la corruzione delle guide spirituali e mostra solo disprezzo per il prestigio economico della sua città, prestigio fondato appunto sull'eccellente credito del fiorino:

La tua città, che di colui è pianta

che pria volse le spalle al suo fattore

e di cui è la ' nvidia tanto pianta, produce e spande il maladetto fiore

c'ha disvïate le pecore e li agni, però che fatto ha lupo del pastore.

[...]

A questo intende il papa e ' cardinali:

(Pd IX 127-136) 


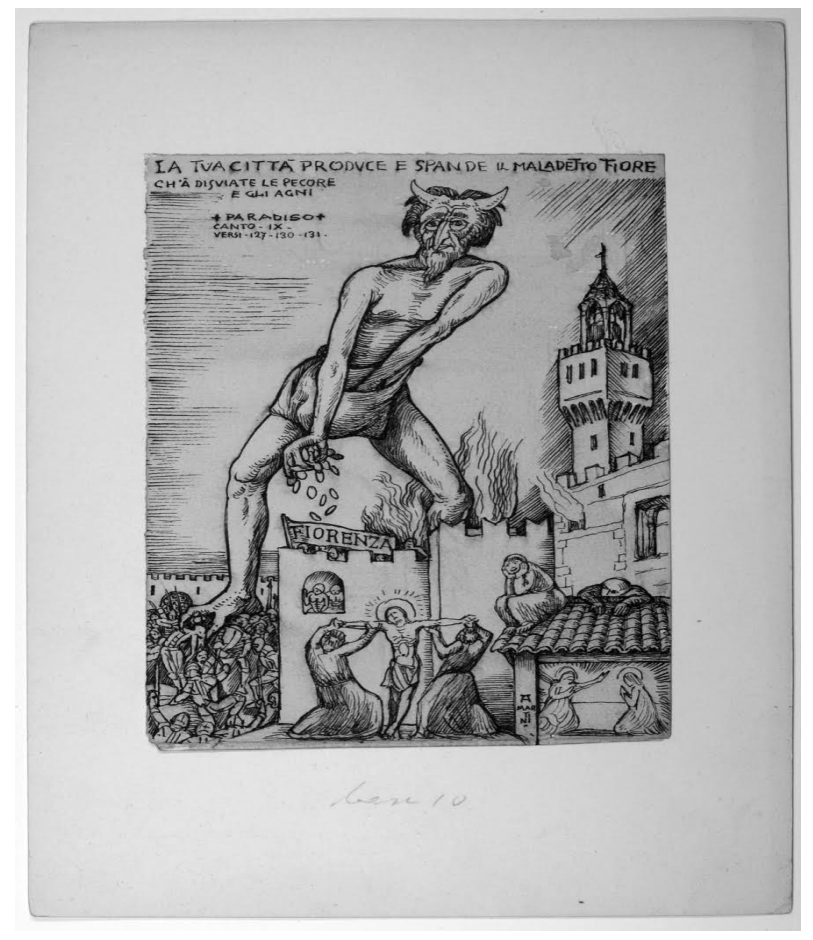

(Fig. 1. Alberto Martini, Paradiso IX. Dalla Collezione della Pinacoteca Alberto Martini, Palazzo Foscolo. Oderzo TV, Italy)

Secondo Forlenza, la ragione del disprezzo di Dante non è solo di natura etica ma anche politica. Nell'agire come depositari delle decime della Chiesa in qualità di banchieri dei papi, i fiorentini trasferivano loro questi proventi in fiorini e i papi a loro volta li distribuivano al clero. I banchieri fiorentini sostenevano anche le attività commerciali lucrative del clero con prestiti, alimentando così ulteriormente la loro cupidigia. ${ }^{8}$ Firenze è rappresentata in questo passo come una pianta diabolica da cui sboccia e si diffonde il maladetto fiore. Come il frutto proibito per Adamo ed Eva, così è questo fiore per Firenze: il suo peccato originale, ciò che la rende città caduta, mondo pastorale sottosopra fondato sull'invidia di Lucifero per i beni goduti dai nostri progenitori nel paradiso terrestre-gli stessi beni da lui perduti dopo la ribellione contro Dio e quella stessa invidia che, come sappiamo dal primo canto del poema, aveva sguinzagliato la lupa fuori dall'Inferno: «là onde 'nvidia prima dipartilla» (If I 111).

${ }^{8}$ Forlenza, Il diritto penale nella Commedia, op. cit., p. 52. 


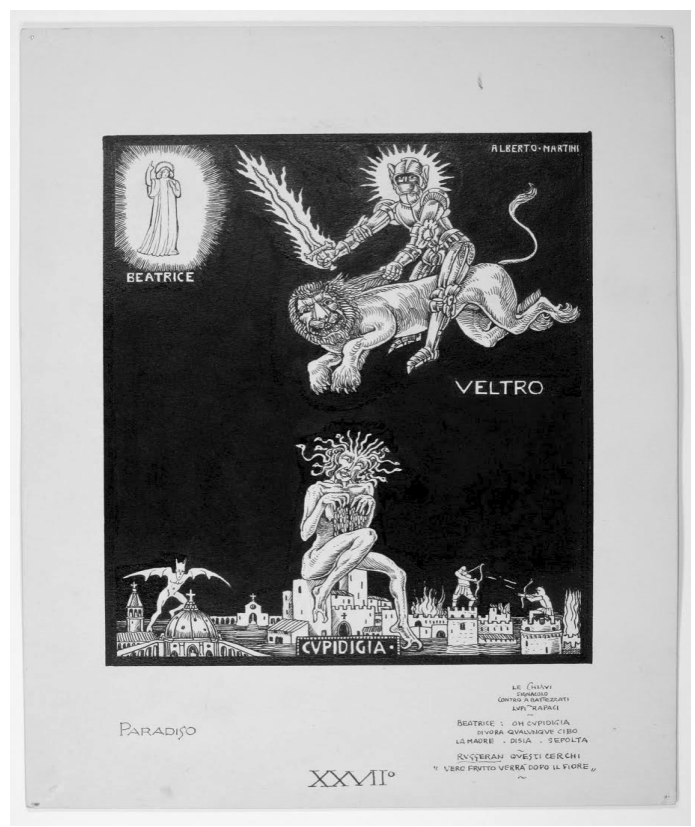

(Fig. 2. Alberto Martini, Paradiso XVIII. Dalla Collezione della Pinacoteca Alberto Martini, Palazzo Foscolo. Oderzo TV, Italy)

L'avidità di denaro dei papi e dei cardinali anticipa il caso di Giovanni XXII il cui fermo disiro per il Battista (Pd XVIII 133-135) allude sarcasticamente all'immagine impressa sul verso del fiorino e il pastore divenuto lupo anticipa l'invettiva di San Pietro contro i lupi rapaci travestiti da pastori (Pd XXVII 55). Ambedue questi momenti ci riportano all'avarizia di tutta la gerarchia ecclesiastica già denunciata nel canto VII dell'Inferno e dei papi in particolare nei canti XIX sia dell'Inferno sia del Purgatorio. L'incipit del passo evoca infine la Firenze di Ciacco, «La tua città, ch'è piena d'invidia sì che già trabocca il sacco» (If VI 49-50), dove la contiguità di superbia, invidia e avarizia (If VI 74) rimanda ancora alla perdita del paradiso terrestre. Per quanto riguarda l'assimilazione dell'avarizia alla cupidigia come radice di ogni male, Dante si avvale di una lunga tradizione, dall'Etica di Aristotele con il commento di San Tommaso, alle Sacre Scritture, agli scritti di Sant'Agostino e a vari testi di carattere moralistico sia d'epoca antica che medievale. ${ }^{9}$ Tuttavia, questo collegamento specifico del fiorino e dell'economia monetaria di Firenze alla Caduta di Adamo, conferma che Dante, reagendo ad una preoccupazione del suo tempo, opera quella «revisione della

${ }^{9}$ Basti vedere questo passo del trattato di Sant'Agostino De libero arbitrio III 48: «Cave enim...id quod dictum est, radicem omnium malorum esse avaritiam (nella fonte-I Tim 6, 10, «radix est cupiditas»)...Haec autem avaritia cupiditas est, cupiditas porro inproba voluntas est». Cfr. Giunta, C. (a cura di), Rime, in Dante Alighieri, Opere, V. 1., C. Giunta, G. Gorni e M. Tavoni (a cura di), Milano, Mondadori, 2001, pp. 558-59; Scott, J., «Avarice in Dante and His Age», Dante Studies. The Annual Journal of the Dante Society of America, 132 (2014), pp. 1-33, alle pp. 1-5. 
gerarchia dei sette peccati capitali» che portò l'«avarizia a spartire con l'orgoglio la posizione di radice o inizio di ogni male» (trad. mia). ${ }^{10}$

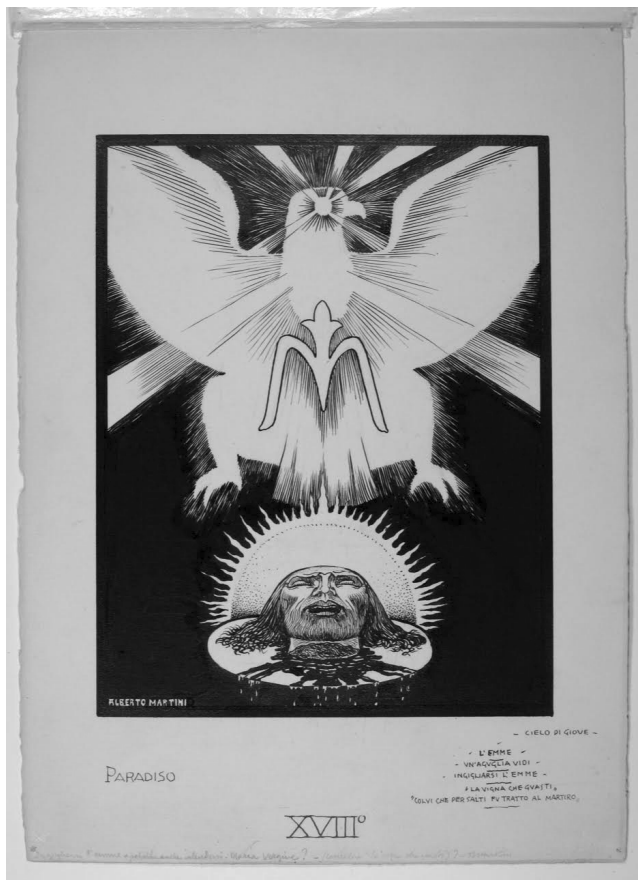

(Fig. 3. Alberto Martini, Paradiso XXVII. Dalla Collezione della Pinacoteca Alberto Martini, Palazzo Foscolo. Oderzo TV, Italy)

Nel quarto trattato del Convivio, Dante affronta il tema della nobiltà inserendo una serie di considerazioni relative alla ricchezza e inizia col confutare l'idea che la nobiltà sia basata su antica ricchezza e belli costumi ( $C V$ IV iii 6), secondo un'opinione attribuita all'Imperatore Federico II. Nell'affrontare questo tema in continuità con la Canzone Le dolci rime, egli dedica vari capitoli (quali xi, xii, xiii) a screditare il fascino de le maladette ricchezze ( $C v$ IV xiii 9), e a dimostrare come esse siano in realtà imperfette, meschine e lontane dalla nobiltà, se non addirittura antitetiche ad essa. Una delle ragioni dell'imperfezione delle ricchezze è, dice Dante, l'«indiscrezione del loro avenimento, nel quale nulla distributiva giustizia risplende, ma tutta iniquitade quasi sempre: la quale iniquitade è proprio effetto

${ }^{10}$ R.A. Shoaf attribuisce tale revisione al "trionfo del denaro», in Dante, Chaucer, and the Currency of the World. Money, Images, and Reference in Late Medieval Poetry, Norman OK, Pilgrim Books, 1983, p. 8. A questo proposito è importante lo studio di L.K. Little: «Se l'orgoglio era il massimo vizio di coloro che detenevano il potere in una società precommerciale, in seguito l'avarizia venne ad occupare una posizione corrispondente rispetto ai ricchi nella società commerciale» (trad. mia), in «Pride Goes Before Avarice: Social Change and the Vices in Latin Christendom», The American Historical Review, 76/1 (1971), pp. 16-49, a p. 38 e n. 89. Le Goff chiama l'orgoglio peccato feudale e l'avarizia, la cui figlia è l'usura, peccato borghese, in J. Le Goff, La borsa e la vita. Dall'usuraio al banchiere, Bari, Laterza, 1987, p. 4. 
d'imperfezione» ( $C V$ IV xi 6). La giustizia distributiva è, secondo Aristotele nel V Libro dell'Etica, la proporzione tra i beni e i meriti individuali. Dante in questo passo non parla del godimento della ricchezza secondo misura, ma piuttosto delle divizie ( $C v$ IV x 7) come false traditrici ( $C V$ IV xii 4), che promettono ingannevolmente l'appagamento mentre, in realtà, facendo aumentare il desiderio-un tipo di desiderio ansioso, quasi una preoccupazione come suggerisce la parola cura (CV IV xii 6)-schiavizzano l'individuo e lo rendono più inquieto e manchevole: «quelle, [...] non solamente non quietare, ma dare più sete e rendere altrui più defettivo e insufficiente» ( $C v$ IV xii 1). Tali ricchezze mettono a repentaglio individui e società: «le cittadi, le contrade, le singulari persone» (CV IV xii 9) e i nuovi desideri generati dalla loro accumulazione non possono essere soddisfatti senza che sia perpetrata ingiustizia verso altri. Le ricchezze stimolano l'avarizia e l'invidia. Invece la virtù della generosità, ovvero della liberalità, alberga in coloro che danno via le ricchezze piuttosto che possederle. ${ }^{11}$ Infine il diritto sia civile che canonico ha la funzione terapeutica di frenare la cupidigia o avarizia generata dall'accumulazione della ricchezza. È chiaro che il leitmotif di queste pagine del Convivio è rappresentato dal binomio ricchezzaingiustizia, tema che è centrale anche nella Monarchia. Il trattato contrappone la cupidigia, o amore 'mal diretto', alla carità intesa nel senso agostiniano di recta dilectio, 'amore retto' nel loro rispettivo rapporto con la giustizia che consiste per Dante in una quaedam rectitudo, 'una sorta di rettitudine', opposta all'obliquità' (Mn I xi 3). La cupidigia, dice Dante, oscura la giustizia, mentre la carità la rafforza (acuit) e la illumina; e la ragione risiede nel diverso obiettivo da esse perseguito: la cupidigia ricerca i beni materiali-e il denaro è certamente tra quelli-alieni dalla natura essenziale dell'essere umano, da ciò che nel linguaggio di Dante è la perseitas hominum, l'impronta del divino, l'imago Dei che è invece l'obiettivo della carità. ${ }^{12}$ Nella Monarchia la nobiltà, diversamente da quello che leggiamo nel Convivio, non è più un valore etico elitario ma diventa valore universale: la possibilità cioè per l'umanità tutta, di recuperare sia con l'intelletto sia con la volontà la similitudine con Dio posseduta da Adamo al momento della creazione ${ }^{13}$. Ed è per questo che, alla fine della Monarchia, Dante rappresenta la felicità terrena che l'umanità può raggiungere con la pratica delle virtù cardinali e intellettuali come una condizione edenica mediante la figura del paradiso terrestre, per terrestrem paradisum figurantur (Mn III Xvi 7).

Nella Commedia, la cupidigia della Monarchia non è altro che la lupa della selva oscura nel primo canto dell'Inferno.

${ }^{11}$ La liberalità è per Dante «the one and only area of wealth-related virtue», 'il solo e unico spazio per la virtú legata alla ricchezza' (trad. mia), in Armour, «Gold, Silver, and True Treasure», op. cit., p. 24.

${ }^{12}$ Stocchi-Perucchio, D., «The Limits of Heterodoxy in Dante's Monarchia», in M.L. Ardizzone (a cura di e con un' «Introduzione»), Dante and Heterodoxy: The Temptation of 13th Century Radical Thought. Cambridge, Cambridge Scholars Publishing, 2014, pp. 197-224, alle pp. 208-209.

${ }^{13}$ Stocchi-Perucchio, D., "Tu l'hai fatto di poco minore che li angeli": Nobility, Imperial Majesty, and the Optimus Finis in Convivio IV and Monarchia», in F. Meier (a cura di), Il Convivio or How to Restart Writing in Exile, Leeds Dante Series, Oxford, Peter Lang, 2018, pp. 225-243. 
Il simbolismo del lupo continua nel VII canto, quello degli avari e prodighi, quando Virgilio mette a tacere Pluto, guardiano del cerchio, chiamandolo maladetto lupo, e poi ancora nel XX canto del Purgatorio dove la lupa riappare come inconfondibile allegoria dell'avarizia:

Maladetta sie tu, antica lupa,

che più che tutte l'altre bestie hai preda

per la tua fame sanza fine cupa!

(Pg XX 10-12)

Maladetta, come le maladette ricchezze del Convivio o il maladetto fiore di Firenze, la lupa annuncia qui l'incontro con Ugo Capeto e il racconto dell'avarizia che aveva caratterizzato questa dinastia regnante. La dimensione politica dell'avarizia è chiara qui come nel primo canto dell'Inferno in cui Virgilio predice l'arrivo del Veltro, allegoria di un imperatore Cristomimete che, portando la giustizia nel mondo, sconfiggerà la bestia. La lupa dantesca che «di tutte brame / sembiava carca ne la sua magrezza» (If I 50) e che "dopo 'l pasto ha più fame che pria» (If I 99), è immagine eloquente di ciò che promette appagamento, come le ricchezze nel Convivio, senza poi mantenere la promessa.

L'allusione alla Caduta di Adamo ricorre chiaramente nel canto XI quando Virgilio, sostando nel cerchio dell'eresia, spiega a Dante la ragione per cui l'usura, come modo aberrante di trattare il denaro, offende la bontà divina:

Ancora in dietro un poco ti rivolvi», diss'io, «là dove di' ch'usura offende la divina Bontade, e 'l groppo solvi». «Filosofia», mi disse, «a chi la 'ntende, nota, non pure in una sola parte, come natura lo suo corso prende dal divino 'ntelletto e da sua arte; e se tu ben la tua Fisica note, tu troverai, non dopo molte carte, che l'arte vostra quella, quanto pote, segue, come 'l maestro fa 'l discente; sì che vostr'arte a Dio quasi è nepote. Da queste due, se tu ti rechi a mente lo Genesì dal principio, convene prender sua vita e avanzar la gente; e perché l'usuriere altra via tene, per sé natura e per la sua seguace dispregia, poi ch'in altro pon la spene.

(If XI 94-111)

Qui Virgilio invoca la Fisica aristotelica e il Genesi. La natura prende il suo corso 
dall'intelletto e dall'arte divina, opera di Dio identificabile con la sua volontà. ${ }^{14}$ L'operosità umana, come insegna Aristotele, imita l'arte divina e cosí si trova in un rapporto genealogico con Dio, dove la natura gli è figlia e l'arte nipote. L'altra analogia è quella del maestro e del discepolo. L'operosità umana imita la natura nella fertilità e produttività allo stesso modo in cui il discepolo imita il maestro. Secondo il Genesi è dunque giusto che gli esseri umani progrediscano e si guadagnino la vita secondo natura e per mezzo dell'arte. L'usura disprezza la natura e l'operosità e così disobbedisce all'ingiunzione fondamentale di Dio fatta ad Adamo ed Eva al momento della cacciata dal paradiso terrestre: «con il sudore del tuo volto mangerai il pane» (3 19), «con dolore partorirai figli» (3 16). ${ }^{15} \mathrm{Il}$ Genesi stabilisce che lavorare e generare figli, cioè produttività e fertilità, definiscono la condizione umana nella storia-da notare a proposito l'affinità semantica tra l'italiano travaglio e lo spagnolo trabajo de parto, come azioni del generare, il francese travailler, 'lavorare' e l'inglese labor, 'lavoro' e 'travaglio'. Nel sistema etico dantesco la sodomia e l'usura-Soddoma e Caorsa (If XI 50)-sono associate tra loro perché entrambe hanno la sterilità come comun denominatore. La sovrapposizione delle due sfere, sessuale ed economica, era luogo comune nel linguaggio giuridico del tempo sia nella legge civile che in quella canonica. Pecunia non parit pecuniam, 'il denaro non genera denaro', era la formula per sanzionare l'illegittimità della produzione di denaro direttamente dal denaro cioè al di fuori del processo produttivo e come frutto del lavoro. ${ }^{16}$ L'usuraio accumula ricchezza mediante l'interesse sul prestito del denaro a così facendo «per se'natura e per la sua seguace / dispregia» (If XI 110-111). Nel condannare l'usura, Dante lancia qui quello che in termini moderni si

${ }^{14}$ A questo proposito cfr. Mn II ii 1-5.

${ }^{15}$ Testo tratto da https://www.maranatha.it/Bibbia/1-Pentateuco/01-GenesiPage.htm.

Data di consultazione 14/11/2019. Tommaso di Chobham, nella sua Summa confessorum, dichiara che «l'usuraio vuol ricavare un profitto senza lavorare affatto e addirittura dormendo, cosa che contravviene al precetto del Signore che dice: 'Con il sudore della tua fronte mangerai il pane'». Cfr. Le Goff, La borsa e la vita, op. cit., p. 36.

${ }^{16}$ La massima ha origine nella Politica di Aristotle, Libro I, 10, 1258b, e si propaga ampiamente nella cultura latina medievale come nummus non parit nummos. Tra le posizioni scolastiche sull'usura cfr. Tommaso D'Aquino, Summa Theologica IIa IIae q. 78, Articolo 1, http://www.corpust homisticum.org/sth3061.html. Data di consultazione 14/11/2019. Cfr. anche Capitani, O., «Sulla questione dell'usura nel Medioevo», in L'etica economica medievale, Bologna, Il Mulino, 1974, pp. 2346; Le Goff, La borsa e la vita, op. cit., p. 19; Amato, M., «Nummus non parit nummos. La questione genealogica dell'uso proprio del denaro», CSV Bergamo 2006, pp. 1-48. https://issuu.com/csvbe rgamo/docs/amato_nummus_non_parit_nummos. Data di consultazione 14/11/2019. Per una rassegna delle posizioni sull'aspetto innaturale dell'usura con la relativa condanna morale dell'interesse nel Giudaismo, nel Cristianesimo e nell'Islam, e sulla questione della sessualità e dell'usura, cfr. Hörisch, J., «Money's Sex», in Heads or Tails. The Poetics of Money, A. Horning Marshall (trad.), Detroit, Wayne State University Press, 2000, pp. 91-126. La prova che il padre di Dante, Alighiero di Bellincione, praticasse l'usura è contenuta in due documenti del 1254 riportati in T. De Robertis, L. Regnicoli, G. Milani e S. Zamponi (a cura di), Codice Diplomatico Dantesco, Roma, Salerno, 2016. Cfr. Armour, sul sonetto ingiurioso di Forese Donati che allude all'attività di Alighiero L'altra notte mi venne una gran tosse, «Gold, Silver, and True Treasure», op. cit., p. 1 e 25 n. 1. 
potrebbe definire un attacco alla logica del profitto come interesse, cioè sterile accumulo di denaro e alienazione degli esseri umani dalla loro condizione esistenziale basata sul lavoro; come trionfo della cupidigia e del narcisismo. Dicendo che l'usuraio pone la sua speranza altrove, Dante implica, per contrasto, che gli esseri umani debbano sperare in un riscatto-Le Goff lo chiama liberazione-in questo caso non in senso trascendentale ma in senso terreno e temporale, raggiungibile mediante il loro impegno nel trasformare e costruire il mondo. Il passare del tempo da cui dipende il guadagno dell'usuraio non è un bene di consumo e quindi non è vendibile come l'usuraio vorrebbe, ma è la dimensione propria in cui questa trasformazione può avvenire. ${ }^{17} \mathrm{Al}$ tempo reificato dell'usuraio Dante contrappone la spinta verso il futuro implicita nella speranza dell'uomo che lavora.

Vorrei suggerire a questo proposito un'analogia tra l'allusione di Dante al potere trasformativo dell'uomo e ciò che Henry chiamerà con Marx-il Marx che egli distingueva vigorosamente dal Marxismo-living labor, 'lavoro vivo'. "The world»scrive Henry-«is only the effect of praxis. The relation to the world through which the world is transformed is a practical relation. [...] Through a set of concepts borrowed from Aristotle, Marx tried to explain how life's hold over things keeps them from nothingness. This hold is living labor [...] Living labor is the ontological operation by which life sustains itself and experiences itself but at the same time sustains everything else that exists» (corsivo originale). ${ }^{18}$ Il linguaggio biblico che Dante usa è distante da quello di Henry ma non per questo meno forte. Vi si avverte che l'ingiunzione divina ai progenitori non è solo una punizione per la loro trasgressione ma il conferimento di un potenziale creativo che diventa un valore etico da proteggere contro ogni aberrazione prodotta dalla violenza o dalla frode.

Nella topografia morale dell'Inferno, l'usura è situata proprio nel cerchio della violenza e al confine con la frode. Sia la violenza che la frode occupano il basso Inferno quali colpe il cui fine è l'ingiustizia. Dante comunica questo criterio a livello teorico, prima che narrativo, per bocca di Virgilio nel canto XI:

D’ogne malizia, ch'odio in cielo acquista,

Ingiuria è 'l fine, ed ogne fin cotale

o con forza o con frode altrui contrista.

(If XI 22-24)

${ }^{17}$ Sul tema della speranza e sull'usuraio come «ladro del tempo», si veda Le Goff, La borsa e la vita, op. cit., pp. 16-17; 27 e seg.

${ }_{18}^{18}$ «l mondo è solo l'effetto della prassi. Il rapporto con il mondo per cui il mondo viene trasformato è un rapporto pratico. [...] Attraverso una serie di concetti tratti da Aristotele, Marx cercò di spiegare come la presa della vita sulle cose le preserva dal nulla. Questa presa è il lavoro vivo [...] Il lavoro vivo è l'operazione ontologica per cui la vita si sostiene e fa esperienza di sè, ma allo stesso tempo sostiene ogni altra cosa che esiste» (trad. mia). In Henry, M., From Communism to Capitalism. Theory of a Catastrophe, S. Davidson (trad.), London \& New York, Bloomsbury, 2014, pp. 65-67. 
Occorre sottolineare qui l'espressione altrui contrista che sembra avere una funzione complementare rispetto alla prima e onnicomprensiva massima morale del poema pronunciata da Beatrice nel secondo canto dell'Inferno:

Temer si dèe di sole quelle cose

c'hanno potenza di fare altrui male;

de l'altre no, ché non son paurose.

(If II 88-90)

La «potenza di fare altrui male» indica che l'alterità è al centro dell'etica dantesca. Lo vediamo anche nella Monarchia quando, nel ribadire che chi amministra la giustizia deve avere «il potere di attribuire a ciascuno ciò che gli spetta» secondo la massima del Digesto (Mn I xi 7), ${ }^{19}$ Dante definisce la giustizia come virtus ad alterum, cioè come virtú sociale in quanto si esercita nei confronti degli altri. Si può dire che questo principio, insieme al concetto della parte equa a cui ciascuno ha diritto in base alla giustizia distributiva, improntano l'insegnamento dantesco sull'economia nel basso Inferno: la logica dell'interesse come egoistico accumulo di plusvalore deve essere corretta con la logica dell'inter-esse, inteso alla latina come 'esistere nel mezzo', ovvero l'intersoggettività che ci caratterizza come esseri sociali. Su questa base si può delineare un'ulteriore analogia con l'inter-esse sul quale si incentra l'ontologia di Levinas e da cui deriva la sua etica del denaro. ${ }^{20}$

Il criterio esposto da Virgilio per la punizione dell'usura nel canto XI dell'Inferno apre la strada alla narrativa vera e propria dell'incontro di Dante con gli usurai nel terzo girone del cerchio della violenza-narrativa che si intreccia con quella del richiamo e poi dell'arrivo di Gerione, la sozza imagine di froda (If XVII 7), il mostro che trasporterà Dante e Virgilio al livello inferiore dell'Inferno. Non c'è da sorprendersi se il cerchio della frode, l'ottavo cerchio detto anche Malebolge, è pieno di peccati collegati al denaro, dall'adulazione e seduzione alla simonia, alla baratteria, al ladrocinio e infine alla falsificazione. Questa gamma di possibilità aberranti è delimitata da due casi specifici sui quali voglio ora soffermarmi. Il primo caso è quello del presta-monete padovano Reginaldo Scrovegni posto nel girone della sodomia e dell'usura; il secondo è quello del falsario Mastro Adamo da Bologna collocato nell'ultima delle Malebolge. Entrambi rappresentano la condizione dell'uomo caduto' senza possibilità di redenzione. Dante ci descrive così la sua ultima sosta nell'arena calda del terzo girone sotto la pioggia di fuoco:

Così ancor sù per la strema testa di quel settimo cerchio tutto solo andai, dove sedea la gente mesta.

${ }^{19}$ Dante Alighieri, Monarchia, A. Tabarroni e P. Chiesa (a cura di), Roma, Salerno, 2013, p. 43.

${ }^{20}$ Levinas, E., «Sociality and Money», in F. Bouchetoux, e J. Campbell (trad.), Business Ethics: A European Review, 16/3 (2007), pp. 203-207. 
Per li occhi fora scoppiava lor duolo; di qua, di là soccorrien con le mani quando a' vapori, e quando al caldo suolo: non altrimenti fan di state i cani or col ceffo or col piè', quando son morsi o da pulci o da mosche o da tafani. Poi che nel viso a certi li occhi porsi, ne' quali 'l doloroso foco casca, non ne conobbi alcun; ma io m'accorsi che dal collo a ciascun pendea una tasca ch'avea certo colore e certo segno, e quindi par che 'l loro occhio si pasca. E com'io riguardando tra lor vegno, in una borsa gialla vidi azzurro che d'un leone avea faccia e contegno. Poi, procedendo di mio sguardo il curro, vidine un'altra come sangue rossa, mostrando un'oca bianca più che burro. E un che d'una scrofa azzurra e grossa segnato avea lo suo sacchetto bianco, mi disse: «Che fai tu in questa fossa? Or te ne va'; e perché sè vivo anco, sappi che 'l mio vicin Vitaliano sederà qui dal mio sinistro fianco. Con questi Fiorentin' son padoano: spesse fiate mi 'ntronan li orecchi gridando: 'Vegna 'l cavalier sovrano, che recherà la tasca con tre becchi!'» Qui distorse la bocca e di fuor trasse la lingua, come bue che 'l naso lecchi.

(IfXVII 43-75)

In questa descrizione colpisce da un lato l'abbondare delle similitudini animalesche-il cane, le pulci, le mosche, i tafani, il bue-che evidenziano il degrado morale di queste anime e dall'altro il reiterato riferimento a singole parti del corpocome il ceffo, il piè, le mani, il muso, la zampa, gli occhi, la bocca, la lingua e il nasoche opera una specie di smembramento figurativo. Quanto agli stemmi che decorano le tasche appese al collo degli usurai, sono anch'essi popolati di animali quali il leone, l'oca, la scrofa, e i becchi, ${ }^{21}$ che si stagliano su una ricca tavolozza di colori araldici. Dal carattere degli stemmi si può evincere che i personaggi a cui gli stemmi appartengono e da cui sono identificati abbiano un pedigree nobiliare di recente acquisizione, non

${ }^{21}$ L'allusione è probabilmente al banchiere Giovanni Buiamonti de' Becchi. Per interpretazioni alternative di questo stemma cfr. Cardini, F., Enciclopedia dantesca, http://www.treccani.it/enciclop edia/giovanni-buiamonti_\%28Enciclopedia-Dantesca\%29/, 1970. Data di consultazione 14/11/ 2019. 
tipico delle famiglie di alto lignaggio ma di quelle di estrazione borghese. Ad ulteriore conferma della tesi del Convivio sulla bassezza delle ricchezze, si tratta qui di una nobiltà devastata piuttosto che sostenuta dal denaro. Gli usurai siedono oziosi a significare il loro disprezzo per la vita activa, mentre i loro occhi cercano di trarre nutrimento e sazietà dalla vista delle borse che pendono loro dal collo, quasi una parodia della vita contemplativa nel suo potere di soddisfare le aspirazioni più alte dell'uomo. ${ }^{22} \mathrm{Il}$ denaro, invece che mezzo di scambio, è divenuto per loro oggetto supremo di desiderio per cui il guadagno materiale si sostituisce a quello che la Monarchia chiamerebbe, come abbiamo già detto, la perseitas hominum ovvero il bene dell'uomo. L'immagine delle parti del corpo che Dante menziona una ad una suggerisce l'idea che l'usura, come vedremo poi anche per la contraffazione del denaro, porti alla disgregazione del corpo politico. Essa implicherebbe infatti un fondamentale disprezzo-che Dante esprime nella Monarchia col verbo sperno (Mn I xi 14)-per il fine ultimo dell'umanità che è quello di ricuperare, con l'aiuto del giusto sistema politico cioè l'impero, l'unità primigenia d'intelletto e di azione sia nella vita contemplativa che in quella attiva. Colui che interloquisce con Dante si può identificare con Reginaldo da Padova grazie alla professata provenienza e allo stemma con la scrofa dipinto sulla sua borsa da cui il cognome Scrovegni. Al tempo della metamorfosi dell'usura in attività bancaria-di cui sono protagonisti gli altri proprietari degli stemmi descritti da Dante quali gli Obriachi, i Gianfigliazzi, i Del Dente e i Buiamonti-Dante deve aver pensato anche ad Enrico Scrovegni, figlio di Reginaldo. Questi fu il ricco banchiere padovano che commissionò a Giotto il famoso ciclo pittorico della Cappella dell'Arena che Dante poté aver visto in fase di esecuzione, forse incontrando lo stesso Giotto di persona. Nel ciclo decorativo, Enrico è ritratto in un angolo del Giudizio Universale nell'atto di offrire la cappella alla Vergine Maria forse nella speranza «di venire / quando che sia a le beate genti» (If I 119-20). È probabile che Dante fosse consapevole, grazie al recente affermarsi della nozione di purgatorio, del fatto che il patronato delle arti e l'ammenda per i peccati commessi andassero di pari passo, pur rimanendo implacabile nella sua condanna dell'usura. ${ }^{23}$

Passiamo ora all'ultima delle Malebolge, le dieci suddivisioni del cerchio della frode, in cui Dante incontra il falsario Mastro Adamo. Questi dichiara di aver

${ }^{22} \mathrm{Si}$ può riconoscere qui un richiamo alla rappresentazione iconografica dell'avarizia quale ritroviamo in un noto bassorilievo nella Chiesa di Santa Maria Assunta a Fornovo (Parma), http://www.luoghimisteriosi.it/emilia_fornovo.html. Secondo J. Scott, Giuda veniva «a volte rappresentato come anima all'Inferno con un una borsa di denaro, simbolo dell'avarizia, ancora appesa al collo» (trad. mia), cfr. «Avarice in Dante», op. cit., p. 4.

${ }^{23}$ Cfr. Le Goff, J., "The Usurer and Purgatory», in The Dawn of Modern Banking, University of California, Los Angeles; Center for Medieval and Renaissance Studies, New Haven CT, Yale University Press, 1979, pp. 25-52; La naissance du Purgatoire, Paris, Gallimard, 1981; «La borsa e la vita. Il Purgatorio», in La borsa e la vita, op. cit., pp., 59-77. Per una sottile lettura della storia di Enrico Scrovegni e la costruzione della Cappella, cfr. Scott, «Avarice in Dante», op. cit., pp. 17-23.

Revista Española de Filosofía Medieval, 27/1 (2020), ISSN: 1133-0902, pp. 121-140 DOI: 
commesso il peccato di contraffazione di denaro per istigazione dei Conti Guidi di Romena. Erano costoro un casato nobiliare del Casentino, valle della Toscana che fu teatro di vari eventi di rilevo nella vita di Dante. Il primo fu certamente la battaglia di Campaldino del 1289 nella quale i Guelfi fiorentini si scontrarono con varie forze Ghibelline tra loro alleate e in cui Dante stesso fu coinvolto come feditore a cavallo. In seguito, un altro ramo della famiglia Guidi ebbe un ruolo importante durante l'esilio del poeta da Firenze in quanto gli dette ospitalità nel proprio castello di Poppi sovrastante la piana di Campaldino, dove pare che egli abbia composto almeno il canto V del Purgatorio. Ecco come Dante ci racconta quest'incontro con Adamo:

Io vidi un, fatto a guisa di lëuto, pur ch'elli avesse avuta l'anguinaia tronca da l'altro che l'uomo ha forcuto. La grave idropesì, che sì dispaia le membra con l'omor che mal converte, che 'l viso non risponde a la ventraia, faceva lui tener le labbra aperte come l'etico fa, che per la sete l'un verso 'l mento e l'altro in sù rinverte. «O voi che sanz'alcuna pena siete, e non so io perché, nel mondo gramo», diss'elli a noi, «guardate e attendete a la miseria del maestro Adamo; io ebbi, vivo, assai di quel ch'i' volli, e ora, lasso!, un gocciol d'acqua bramo. Li ruscelletti che d'i verdi colli del Casentin discendon giuso in Arno, faccendo i lor canali freddi e molli, sempre mi stanno innanzi, e non indarno, ché l'imagine lor vie più m'asciuga che 'l male ond'io nel volto mi discarno. La rigida giustizia che mi fruga tragge cagion del loco ov'io peccai a metter più li miei sospiri in fuga. Ivi è Romena, là dov'io falsai la lega suggellata del Batista; per ch'io il corpo sù arso lasciai. Ma s'io vedessi qui l'anima trista di Guido o d'Alessandro o di lor frate, per Fonte Branda non darei la vista.

[...]

Io son per lor tra sì fatta famiglia;

e' m'indussero a batter li fiorini ch'avevan tre carati di mondiglia».

(If XXX 49-92) 
La condanna di Mastro Adamo ha un doppio aspetto, fisico e psicologico. Il suo corpo è deformato da uno smisurato gonfiore e dai fluidi che gli stagnano dentro, mentre la sua mente è ossessionata dalla visione dei ruscelli del Casentino e dal ricordo di quelle fresche acque correnti che serve ad acuire in lui la tortura della sete. La deformità di Mastro Adamo rappresenta la versione distorta della perfezione del primo uomo, di cui egli porta il nome, creato a immagine e somiglianza di Dio, mentre gli ameni corsi d'acqua del Casentino sembrano alludere, in negativo, alla fluidità della materia fusa strumento del falsario e, in positivo, ad un paradiso perduto e ad un'acqua battesimale istituita al fine di capovolgere l'effetto del peccato originale. ${ }^{24}$ L'episodio di Adamo non finisce qui ma seguita con un rauco e violento scontro verbale pieno di insulti reciproci scambiati con Sinone di Troia, un altro peccatore che condivide con Adamo la stessa dimora infernale. Queste sono le ultime parole che Adamo gli scaglia contro:

\section{[...] s'i' ho sete e omor mi rinfarcia, tu hai l'arsura e 'l capo che ti duole, e per leccar lo specchio di Narcisso, non vorresti a 'nvitar molte parole».}

(If XXX 126-129)

Dante non potrebbe essere più esplicito. Narciso, che rappresenta l'amore per se stessi, fa pensare qui all'interesse, al beneficio del 'sé' che la falsificazione comporta. I due personaggi esemplificano insieme una comunità disfunzionale e malata che questo tipo d'interesse genera. Sono immagini speculari; il loro rispettivo narcisismo impedisce ad entrambi di vedere l'altro come altro; sono entrambi ciechi all'alterità dell'inter-esse, come essere 'fra'. ${ }^{25}$

È per questo che le cose di cui v'è scambio devono essere in qualche modo commensurabili. [20]»-leggiamo nel libro V dell'Etica a Nicomaco-《A questo scopo è stata introdotta la moneta, che, in certo qual modo, funge da termine medio: essa, infatti, misura tutto, per conseguenza anche l'eccesso e il difetto di valore [...] Infatti, se questo non avviene, non ci sarà scambio né comunità [la parola greca è koinonia. Inciso mio]. [25] E questo non si attuerà, se i beni da scambiare non sono in qualche modo uguali. Bisogna, dunque, che tutti i prodotti trovino la loro misura in una sola cosa, come abbiamo detto prima. E questo in realtà è il bisogno, che unifica tutto: se gli uomini, infatti, non avessero bisogno di nulla, o non avessero gli stessi bisogni, lo scambio non ci sarebbe o non sarebbe lo stesso. E come mezzo di scambio per

\footnotetext{
${ }^{24}$ Cfr. Mussetter, S.A., «Inferno XXX: Dante's Counterfeit Adam», Traditio, 34 (1978), pp. 427-435.

${ }^{25} \mathrm{Il}$ narcisismo come comun denominatore sia dell'usura che della falsificazione emerge anche dall'osservazione di J. Hörisch che il ventre gonfio e tondeggiante di Mastro Adamo «fa diventare il falsario e ri-produttore di denaro la caricatura di una donna gravida». Egli aggiunge anche che «il romanzo di Gide I falsari, riprende questo motivo dantesco e mette abilmente in luce la componente omoerotica della riproduzione del denaro, ovvero della falsificazione» (trad. mia), in Heads or Tails, op. cit., p. 107. Sul narcisismo cfr. anche «Narcissus Damned, or the Failure of Reference (Inferno 30)», ibid., pp. 39-48.
} 
soddisfare il bisogno è nata, per convenzione, la moneta. [30] E per questo essa ha il nome di novmisma [moneta], perché non esiste per natura ma per 'nomo' [legge]. ${ }^{26}$

La falsificazione di Adamo de la lega suggellata del Batista, nell'alterare l'unità di misura con una carenza-i tre carati di mondiglia-ha violato la convenzione che rende possibile la corretta e giusta proporzione dello scambio reciproco e che, secondo Aristotele, mantiene coesa la comunità. Rivisitando implicitamente lo stesso passo Aristotelico, Levinas, nel suo saggio «Sociality and Money», 'Socialità e denaro' afferma che «il plusvalore minaccia la misurazione esatta del valore del lavoro». ${ }^{27} \mathrm{Di}$ fronte a questo ci si chiede se l'autore de L'Ottimo Commento alla Divina Commedia, scritto nel 1338, non pensasse negli stessi termini mentre glossava il passo sull'usura nel canto XI dell' Inferno dicendo, sempre con Aristotele, che il denaro era stato inventato per eguagliare cose ineguali: «come dice il Phylosopho nel .v. de l'Ethica, «lo danaio fu trovato primamente per aguagliamento delle cose ch'erano disiguale». ${ }^{28}$ Dante ritiene Mastro Adamo ovviamente responsabile di un reato che mette a repentaglio la coesione della comunità, ma non manca di sottolineare anche la responsabilità degli istigatori i cui motivi andavano forse al di là del puro guadagno personale. Con ogni probabilità-ed è questa l'ipotesi di Forlenza ${ }^{29}$-c'era in gioco la rivalità tra questi signori ghibellini e la Repubblica Fiorentina che a quel tempo era sotto controllo guelfo. Non essendo in grado di prevalere sul campo di battaglia, i Guidi erano ricorsi ad una specie di guerra economica per danneggiare l'attività commerciale e bancaria di Firenze. È così che la prospettiva filosofica si combina con la concreta realtà politica della guerra civile. Oltre al caso di Mastro Adamo e dei Conti Guidi, Dante sceglie altri due esempi di uso scorretto del denaro entrambi per mano di sovrani. Il contesto è il cielo di Giove, dedicato alla Giustizia, in cui Dante elenca tutte le malefatte dei sovrani cristiani d'Europa. Il primo è Filippo il Bello che alterò il valore della moneta francese-falseggiando la moneta (Pd XIX 119)-coniando cioè monete il cui valore reale era inferiore al valore nominale per finanziare la guerra contro le Fiandre. Dal momento che battere moneta era prerogativa imperiale e reale e il sovrano era il garante dell'autenticità, la gravità di questa trasgressione era agli occhi di Dante molto superiore a quella commessa da un individuo comune dal momento che ad essere minacciata era la comunità in senso lato ovvero la Francia tutta. L'altro caso è quello del re di Serbia Stefano II che, nello stesso contesto dei sovrani corrotti, falsificò il ducato veneziano e lo mise in circolazione allo scopo di causare un danno economico a Venezia-«che male ha visto il conio di Vinegia» (Pd XIX 141).

${ }^{26}$ Aristotele, Etica a Nicomaco V, http://www.filosofico.net/eticaanicomaco5.htm. Data di consultazione 14/11/2019.

${ }^{27}$ Il passo completo recita: «But by the measure that it introduces everywhere, exchangefrom which money emerged-is freed from the confusion and subjective influences of barter: with the arrival of money, 'goods' constitute an objective whole despite-needless to say-the unexpected way that surplus value-as it was later called-threatens the exact measurement of the value of labor», in «Sociality and Money», op. cit., p. 204.

${ }^{28}$ L'Ottimo Commento, https://dante.dartmouth.edu/search_view.php?doc=133371110970\&c md=gotoresult\&arg1=5. Data di consultazione 14/11/2019.

${ }^{29}$ Forlenza, Il diritto penale nella Commedia, op. cit., p. 55. 
Queste connessioni mettono in evidenza la contiguità dell'usura e della falsificazione e aprono lo spazio del confronto con le realtà contemporanee. Il prestamonete Reginaldo Scrovegni potrebbe essere l'antecedente di certi odierni padroni della finanza mondiale ${ }^{30}$ mentre il falsario Mastro Adamo potrebbe anticipare quei contemporanei operatori finanziari e quelle banche che contraffanno valori e manipolano valute, come nel caso del recente scandalo Libor, alla ricerca di profitti e senza curarsi minimamente delle conseguenze per il prossimo, vicino o lontano, sia che si tratti di singoli individui che di intere popolazioni-i cosiddetti terzi, 'the third parties' di cui parla Levinas (2007: 206). ${ }^{31}$

(Fig. 4. Luis Agusti, Dante vivo. Reflexiones a partir del Infierno. Séptimo círculo (tercer jirón): Violentos contra Dios (usureros) 2000.

Ensamblaje: cadenas, fierro, corian, MDF, autoahesivo 175 x 40 x $40 \mathrm{~cm}$. Colección Micromuseo, Lima)

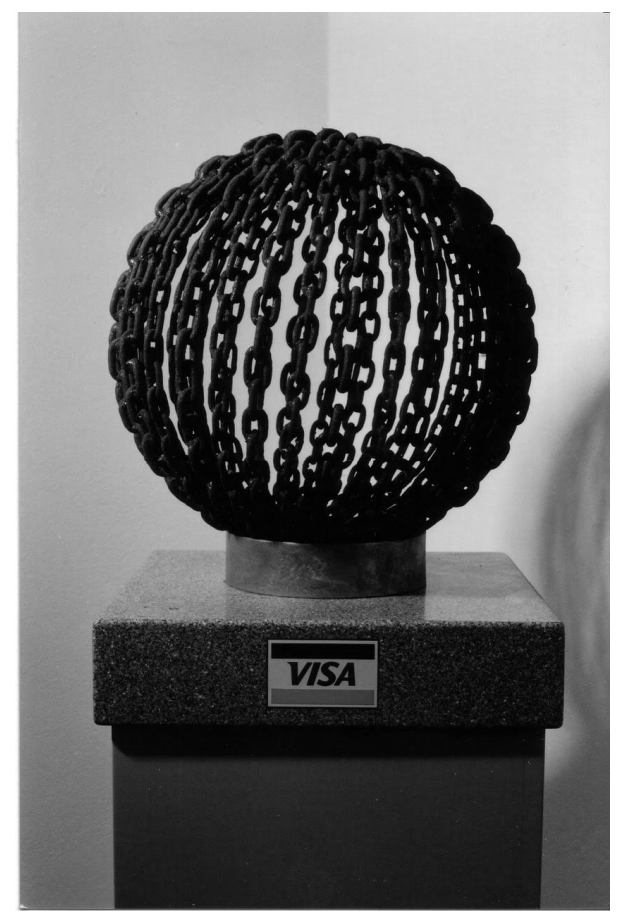

${ }^{30} \mathrm{Cfr}$. Arlacchi, P., I padroni della finanza mondiale: Lo strapotere che ci minaccia e i contromovimenti che lo combattono, Milano, Chiarelettere, 2018.

${ }^{31}$ Cfr. McBride, J., «Understanding the Libor Scandal», Council on Foreign Relations. Last updated October 12, 2016.

https://www.cfr.org/backgrounder/understanding-libor-scandal. Data di consultazione 14/11/2019; Levinas, «Sociality and Money», op. cit., p. 206. Cfr. anche «Our Take on the 10 Biggest Frauds in U.S. History», Forbes, https://www.forbes.com/pictures/efik45ekdjl/our-take-on-the-10biggest-frauds-in-recent-u-s-history-2/\#62ada796775a. Data di consultazione 14/11/2019; e «Ten Financial Frauds that Shook the World», The Economic Times, https://economictimes.indiatimes.co $\mathrm{m} / 10$-financial-frauds-that-shook-the-world/articlelist/3847076.cms, 16/12/2008. Data di consultazione $14 / 11 / 2019$. 
È risaputo che Dante riserva la più grande ammirazione per il modello assoluto di Francesco d'Assisi che sfida i valori economici del suo tempo rifuggendo del tutto non solo il denaro ma qualsiasi altra forma di proprietà materiale..$^{32}$ Allo stesso tempo, teorizzando una via politica per costruire la giustizia terrena nella Monarchia, e rappresentando una gamma di comportamenti aberranti verso il lavoro e la produttività nella Commedia, Dante ci sfida ad immaginare, per contrasto, il mondo come campagna felix, 'campagna felice' in cui fertilità e felicità coincidono ${ }^{33}$ In questa luce, la beatitudo huius vitae, 'la felicità di questa vita' della Monarchia, emerge come prodotto dell'umano potere generativo attraverso cui, imitando la natura come arte di Dio, l'umanità plasma la storia e trasforma il mondo attuando così il principio della sua creazione come imago Dei.

Donatella Stocchi-Perucchio donatella.stocchi-perucchio@rochester.edu

Fecha de recepción: 03/11/2019

Fecha de aceptación: 02/03/2020

${ }^{32}$ È vero che proprio dalle fila dagli ordini mendicanti-i quali teorizzavano e praticavano la povertà volontaria, come Pietro di Giovanni Olivi e Remigio de Girolami-vediamo emergere un nuovo concetto di profitto che, se basato sulla compartecipazione e l'equità, serve il bene comune. Resta da vedere fino a che punto Dante riconosca quest'alternativa positiva come concreta realtà del suo tempo. Cfr. Todeschini, F., Ricchezza francescana. Dalla povertà volontaria alla società di mercato, Bologna, Il Mulino, 2004; Kaye, J., A History of Balance, 1250-1375. The Emergence of a New Model of Equilibrium and its Impact on Thought, Cambridge, UK, Cambridge University Press, 2014.

${ }^{33}$ Ringrazio qui il Professor Carlos Gatti-Murriel dell'Università del Pacifico di Lima, Perù, per ricordarci sempre nelle sue lezioni la saggezza del latino. A proposito, sarà bene ricordare anche la saggezza dell'ebraico dove 'éden «in origine significò 'campagna', e poi anche 'piacere, delizia'». Vocabolario Treccani, http://www.treccani.it/vocabolario/eden/. Data di consultazione 13/7/2020. 